\title{
Automatic image processing in developmental testing of visual-motor integration
}

\author{
M.C.Fairhurst, N.Higson, C.Clar \\ Electronic Engineering Laboratories, University of Kent, \\ Canterbury, Kent CT2 7NT, U.K.
}

R.Bradford, W.Clark, E.Pringle

Mary Sheridan Centre, Kent and Canterbury Hospitals NHS Trust, 43, New Dover Road, Canterbury, Kent CT1 3AT, U.K.

\begin{abstract}
Figure copying and related tasks are commonly used in the evaluation of perceptual and motor-perceptual functioning, yet there are many potential difficulties associated with the administration of such tests and their objective assessment. This paper describes a study, based on the Beery Developmental Test of Visual-Motor Integration, which investigates the use of automatic image analysis techniques to implement and evaluate figure copying tasks, and which can afford the opportunity for the extraction of further characteristics of drawing execution which are of potential significance in assessing performance.
\end{abstract}

\section{Background}

The evaluation of neurological functioning is important in the monitoring and assessment of a variety of clinical conditions, and a range of cognitive and perceptual tests have been developed over the years in order to achieve this $[1,2,3,4,5]$. The assessment of perceptual and motor-perceptual ability in children with learning difficulties has in recent years been accorded a higher priority than hitherto with the increasing awareness of the problem of dyspraxia, the incidence rate of which is estimated to be of the order of 6-7\%, and may be greater [6].

Although many forms of testing may be considered in investigating perceptualmotor functioning, the difficulties in applying many traditional testing procedures generally relate to the severe demands they place on available resources, both in terms of the time taken for their administration and/or the requirement for specially trained clinical staff, the stress often induced in the patient by the procedures involved, and the unsuitability of the tests for regular or repeated performance. Even more importantly, the evaluation of test results can often be very subjective and inherently unreliable. Figure copying and related tasks in particular, however, 
suggest an approach to the evaluation of perceptual and motor-perceptual ability which is potentially very suitable for computer-assisted implementation and which might be able to provide interpretation on a widely accepted basis [7].

This paper will describe an approach to perceptual testing which utilises image analysis techniques to provide the framework for the implementation of a computerbased testing and evaluation system suitable for routine clinical use. The results of some initial experimentation with a prototype system are described and discussed.

\section{Developmental testing}

A widely used test adopted for developmental assessment of children is the Beery Developmental Test of Visual-Motor Integration [8]. This test has been designed to assess the developmental maturity of 5 to 11 year old children in relation to their visual, fine-motor and visual-motor development.

The test consists of 24 geometric shapes of increasing complexity, starting with single lines and simple shapes such as the circle, square and triangle, and proceeding to more complex combinations of shapes and three-dimensional representations of shapes. The shapes are displayed on the top half of a sheet of paper (three shapes to a page) and have to be copied by the child in boxes underneath the target shapes. The copying task requires the child to combine his/her ability accurately to perceive a given form with the ability accurately to reproduce it, and hence is an indicator of visual-motor integration. However, it also allows the identification of children with pure perceptual or pure fine-motor difficulties - a child drawing confidently but inaccurately may be suspected to have principally perceptual problems, whereas a child obviously struggling with the execution of the drawing but giving other evidence of correctly perceiving the shape will be suspected to have mainly finemotor difficulties.

The shapes comprising the test were originally chosen on the basis of large studies of children's copying behaviour, and a review of their ability in drawing and copying as determined through studies of the literature. These studies were also the origin of the establishment of age-equivalents for each score, giving an indication of which shapes a child should be able correctly to reproduce at a given age.

The individual shapes are scored according to strictly defined criteria in an all-ornothing manner (i.e. no partial marks are given). The scoring is graded according to difficulty (i.e. score 1 or 0 for the easiest shapes, up to 4 or 0 for the most difficult), and a composite score is calculated at the end and converted to an age-equivalent score.

Although a widely used and well established standard test, the Beery test is nevertheless vulnerable to many of the difficulties outlined above. In particular, questions concerning the issue of objectivity in scoring (allowing for inconsistencies 
in the interpretation of the scoring rules) and the problems of administering and scoring the test, detract from its overall effectiveness as an evaluative tool.

For these reasons, work is in progress to investigate the use of automated evaluation of testing procedures such as that instantiated by the Beery test. The aim is to use computer-based acquisition and analysis of the test data and the subsequent automated scoring of the test results. In this way, the power of computer-based processing can be exploited to remove many of the difficulties encountered with this type of testing and, as will be seen, can also potentially enhance the effectiveness and diagnostic capabilities of the testing procedure.

\section{The testing environment}

The task domain requires a subject to generate line drawings (by copying target shapes) which are subsequently to be analysed according to pre-defined scoring rules. In order to enable computer-based acquisition of the drawing the target shape is copied by the subject on a graphics tablet which is interfaced to a microcomputer. This allows the acquisition of the drawing data as a stream of coordinate mappings, and the analysis of this data, in real time. The general system infrastructure is illustrated in Figure 1.

\section{Figure 1}

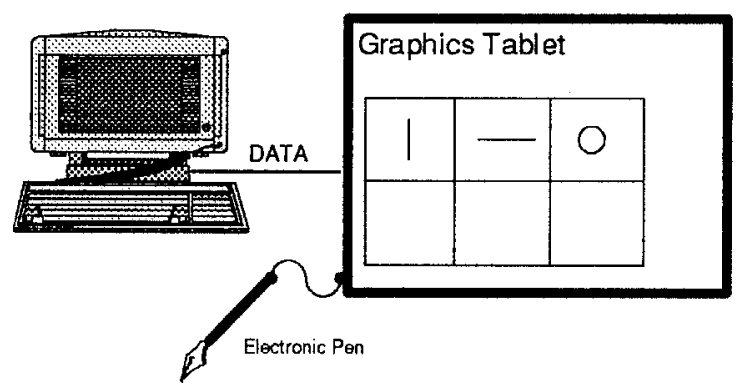

Figure 1

A principal advantage of this data acquisition environment is that, although complete data capture can be readily achieved, there is no interference from the subject's viewpoint with the normal procedure involved with standard pencil-andpaper execution of the test. The system uses an electronic pen which can also 
generate hard copy in the traditional way. The pen may be an integral wired-in device or, depending on the choice of tablet, can be wire-free, and the task execution is thereby achieved in an entirely familiar fashion.

\section{Preliminary results}

Initial trials have been carried out to establish the viability of the approach proposed. The complexity of the geometric shapes to be analysed varies considerably, becoming progressively more complex as the test proceeds. Figure 2 shows the first and last shapes in the sequence to illustrate this point.

Figure 2

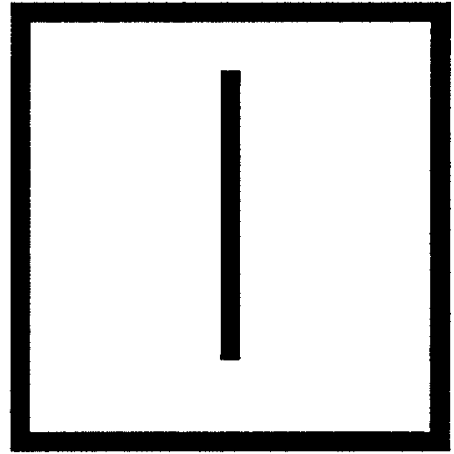

1st Shape

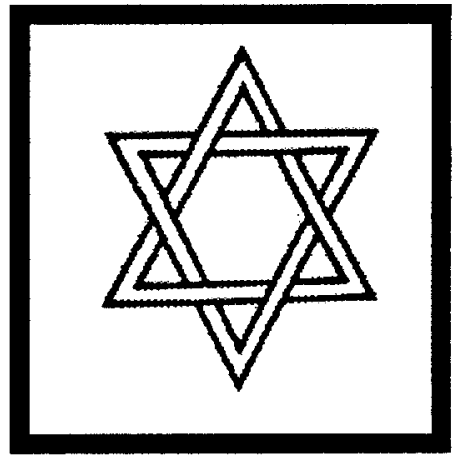

Last Shape

Appropriate image processing algorithms have been designed to analyse the drawings produced and recorded during computer-based administration of the test. For some shapes these require the direct extraction of relevant features from the images produced, while for the more complex shapes more extensive knowledgedriven procedures for interpretation of the defined scoring rules are required.

A test was carried out in which 15 children (aged between 5 and 12 years, and referred to an assessment centre dealing with learning and coordination difficulties) were tested with the system, their drawing data being acquired on-line while hard copies of the test output were simultaneously obtained. The test output was then scored both by the occupational therapist administering the test (from the hard copy output) and by means of the computer-based automated implementation of the scoring rules (from the tablet data).

Figure 3 shows the results obtained in initial testing. It can be seen that the majority of target shapes show a complete convergence between automated scoring and the human expert scorer. The discrepancies observed in some instances are due largely 
to the current lack of sufficient data to allow adequate implementation of the scoring rules (this is the case, for example, with shape 23), while in other cases the variability of the data obtained leads to atypical or unpredictable patterns, a situation which requires refinement of the scoring algorithms to increase their robustness. Current work is seeking to refine and enhance the algorithms to improve their sensitivity, power and reliability in dealing with such problem cases.

\section{Figure 3}

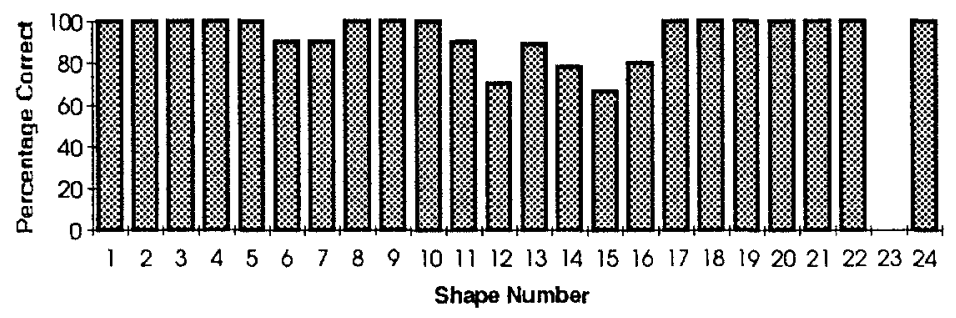

\section{Further refinement of computer-based testing}

Although a principal aim of the work reported here is to increase efficiency and objectivity of testing through the automation of evaluative procedures, a further potentially highly significant benefit arising from the exploitation of the automated testing environment described here should be noted. In its present (pencil-andpaper) form the test focuses on the extraction of information from each test sample only of static features of the test drawing, features related to the finished drawing and observable solely from the overall visual appearance and broad characteristics of the child's output. Related research reported in recent years [9] strongly suggests that further very important information about performance is likely to be found in the dynamic features of the output, features which characterise specifically the actual physical execution of the drawing. In particular, the dynamic information gathered may allow a more detailed analysis of the mechanisms of a given child's impairment. Although this is the subject of continuing research, it is valuable to indicate an example of how such additional information might be considered.

Since the computer-based data acquisition environment allows the on-line monitoring of task execution, information about dynamic characteristics such as pen velocity can be readily measured. In order to demonstrate the potential value of such measurements, Figure 4 illustrates the use of pen velocity profiles, showing the mean score obtained by the five fastest drawers, the five slowest drawers, and the five drawers exhibiting intermediate velocity profiles in the tests described above. Although no formal analysis has been carried out here, it is nevertheless possible to hypothesise that very low pen velocities correlate with difficulties in drawing execution and that very high pen velocities are indicative of difficulties in 
coordination or carelessness (thereby generating lower age-equivalent scores), while an appropriately balanced velocity profile correlates with higher levels of achievement.

\section{Figure 4}

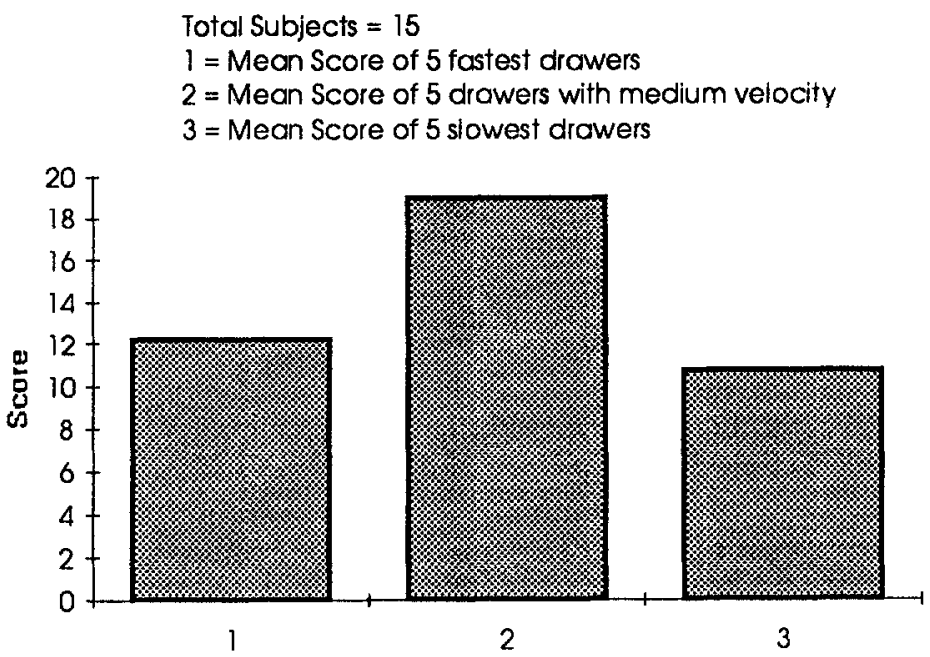

There are clearly also many other such dynamic factors which may prove to be relevant and important, particularly those concerned with constructional strategy (stroke sequencing, directionality, and so on) or other behavioural characteristics. Issues such as this clearly require more thorough investigation, but do appear to point to the potential value of the principle of extracting hitherto unobservable performance characteristics such as those related to dynamic execution.

\section{Conclusion}

This paper has described a new approach to the administration of standard testing procedures for the evaluation of perceptual and motor-perceptual functioning. The testing procedures proposed exploit computer-based image processing techniques to improve objectivity and efficiency in the testing process while, as an added advantage, offering the possibility of allowing the extraction of additional dynamic characteristics of performance which might provide further important diagnostic information.

Although only a preliminary study is reported here, the results presented are most encouraging, justifying the further work which is presently in progress. 


\section{References}

[1] Anastasi,A.: Psychological Testing, Macmillan, New York, 1968

[2] Ackrill,P., Barron,J. et al: A new approach to the early detection of dialysis encephalopathy", Proc. EDTA, 16, 659-660, 1979

[3] Miller, E.: Abnormal Ageing, John Wiley and Sons, London, 1977

[4] Pearce,J. and Miller,E.: Clinical Aspects of Dementia, Bailliere Tindall, London, 1973

[5] De Renzi,E.: Disorders of Space Exploration and Cognition, John Wiley and Sons, New York, 1982

[6] Brenner et al: British Medical Journal, (iv), 259-262, 1967

[7] Ilg and Ames: Child Behaviour, Harper, New York, 1955

[8] Beery,K.E.: The VMI-Developmental Test of Visual-Motor Integration Administration, Scoring and Teaching Manual, 3rd Revision, Cleveland: Modern Curriculum Press, 1989

[9] Fairhurst,M.C., Smith,S.L. and Potter,J.: Computer-assisted analysis of visuospatial neglect in stroke patients, Proc. 3rd Int. ICCHP Conf., 157-165, Vienna, July 1992 